\title{
Análisis de redes sociales de la producción científica sobre programación televisiva
}

\author{
Analysis of social networks of scientific production on television \\ programming
}

\begin{abstract}
Análise de redes sociais de produção científica em programação televisiva
\end{abstract}

\author{
Alicia Moreno-Delgado \\ Estudiante de doctorado \\ (Universidad Internacional de La Rioja, UNIR) \\ https://orcid.org/0000-0002-3425-061X \\ España \\ Dr. Rafael Repiso \\ Profesor titular \\ (Universidad Internacional de La Rioja, UNIR) \\ https://orcid.org/0000-0002-2803-7505 \\ España \\ Dr. Julio Montero-Díaz \\ Vicerrector de Comunicación \\ (Universidad Internacional de La Rioja, UNIR) \\ https://orcid.org/0000-0002-4145-7424 \\ España
}

Fecha de recepción: 27 de noviembre de 2019

Fecha de revisión: 28 de noviembre de 2019

Fecha de aceptación: 15 de diciembre de 2019

Fecha de publicación: 1 de enero de 2020

Para citar este artículo: Moreno-Delgado, A., Repiso, R. y Montero-Díaz, J. (2020). Análisis de redes sociales de la producción científica sobre programación televisiva, Icono 14, 18 (1), 123-154. doi: 10.7195/ri14.v18i1.1484 


\section{Resumen}

Se analiza la producción académica indexada en WoS sobre la programación televisiva. Se lleva a cabo un análisis diacrónico que estudia la evolución de los términos empleados en cada etapa y su clusterización para ofrecer una primera base de las preocupaciones de los investigadores académicos sobre la programación televisiva y sus efectos. Se atiende a las redes identificadas entre los trabajos académicos. Los objetivos planteados son: determinar las áreas de conocimiento donde se publican los trabajos sobre Programación de Televisión; identificar los documentos más relevantes; identificar los temas predominantes. Se utiliza una metodología original partiendo de un conjunto de datos de Web of Science (WoS) y, mediante una técnica de recopilación de bola de nieve, se registran los trabajos más significativos incluyendo aquellos externos a WoS. Se aplican dos tipos de análisis de contenido con un fuerte componente automatizado: Análisis Bibliométricos y Análisis de Redes Sociales a documentos científicos a partir los registros de WoS Core Collection. Los resultados muestran cómo el área de Comunicación y el de Cine, Radio y Televisión conforman más del 50 por ciento del total de la producción científica. Además, se ha identificado que el artículo más relevante pertenece al área de Comunicación el segundo y tercer documento con más citas son publicaciones del ámbito de la Medicina. En cuanto a la temática, existen dos áreas: la primera estudia la programación televisiva de forma directa: historia y evolución, competencia, contenidos y publicidad; la segunda centrada en el análisis de la influencia de la programación en otras dimensiones: obesidad, violencia, racismo y estereotipos.

Palabras clave: Programación; Televisión; Programación televisiva; Producción científica; Bibliometría; Redes sociales

\section{Abstract}

The academic production indexed in WoS on television programming is analyzed. A diachronic analysis is carried out that studies the evolution of the terms used in each stage and their clustering in order to offer a first step of the academic researchers' concerns about television programming and its effects. The networks identified among the academic works are taken into account. The proposed objectives are: to determine the areas of knowledge where the works on Television Programming are published; to identify the most relevant 
documents; to identify the predominant topics. We use an original methodology based on a Web of Science (WoS) data set and, through a snowball compilation technique, we record the most significant works including those external to WoS. There are two types of content analysis with a strong automated component: Bibliometric Analysis and Social Network Analysis are applied to scientific documents from the WoS Core Collection records. The results show how Communication and Film, Radio and Television make up more than 50 percent of total scientific production. In addition, it has been identified that the most relevant article belongs to the area of Communication, the second and third documents with the most citations are publications in the field of Medicine. Regarding the topic, there are two areas: the first focuses on television programming itself: history and evolution, competition, content and advertising; the second focuses on the analysis of the influence of programming on other dimensions: obesity, violence, racism and stereotypes.

Key Words: Programming; Television; TV programming; Scientific production; Bibliometrics; Social networks

\section{Resumo}

A produção acadêmica indexada na WoS na programação de televisão é analisada. $E$ realizada uma análise diacrónica que estuda a evolução dos termos utilizados em cada etapa e o seu agrupamento para formecer uma base inicial para as preocupações dos investigadores académicos sobre a programação televisiva e os seus efeitos. A atenção é dada às redes identificadas entre os trabalhos acadêmicos. Os objectivos são: determinar as áreas de conhecimento onde os trabalhos sobre Programação Televisiva são publicados; identificar os documentos mais relevantes; identificar os temas predominantes. Uma metodologia original é utilizada com base num conjunto de dados da Web of Science (WoS) e, através de uma técnica de recolha de bolas de neve, são registados os trabalhos mais significativos, incluindo aqueles externos à WoS. Dois tipos de análise de conteúdo com um forte componente automatizado são aplicados: Análise Bibliométrica e Análise de Redes Sociais a trabalhos cientificos dos registros da Coleção WoS Core. Os resultados mostram como a área da Comunicação e a do Cinema, Rádio e Televisão constituem mais de 50\% do total da produção científica. Além disso, foi identificado que o artigo mais relevante pertence à área da Comunicação o segundo e terceiro documentos com mais citações são publicações na área da Medicina. Em relação ao tema, há duas áreas: a primeira estuda diretamente 
a programação televisiva: história e evolução, competição, conteúdo e publicidade; a segunda focaliza a análise da influência da programação em outras dimensões: obesidade, violência, racismo e estereótipos.

Palavras chave: Programação; Televisão; Programação televisiva; Produção científica; Bibliometria; Redes sociais

\section{Introducción y estado de la cuestión}

Los estudios sobre programación televisiva solo constituyen una de las posibles líneas de investigación sobre este medio (Bignell, 2012; Brunsdon, 1998; Turner \& Tay, 2009). Sin embargo, la programación es el elemento esencial de la televisión anterior a la oferta televisiva a la carta. Construye precisamente esa conversación que cada cadena mantiene con sus espectadores (Williams, 1992; Corner, 1999; Fiske, 1987; para el caso español Montero-Díaz, 2014 y Montero-Díaz, 2018). Concreta en una oferta cerrada, completa y articulada lo característico del flow televisivo de cada cadena en su doble sentido: como fuerza que arrastra en un determinado sentido los contenidos y los contenidos mismos que lo componen (Williams, 1992). Por eso, el análisis de la producción académica sobre programación televisiva apunta a un elemento esencial en esta área y articula, de algún modo, los estudios sobre televisión. Tomarlo como elemento de referencia clave de este análisis no responde, por tanto, a una decisión arbitraria.

Sensu contrario, el prescindir de la programación en los estudios de televisión, llevaría a reducirla a un elemento puramente técnico: una pantalla de dimensiones medias, fija, de la que puede disfrutarse en el hogar, sin modificaciones en la estructura arquitectónica. En definitiva, un electrodoméstico más de indudable utilidad: un terminal para el consumo inmediato de productos audiovisuales variados que se solicitan a la carta al almacén que los conserva y que se sirve de modo inmediato. No es extraño que Amazon sienta como propia esta actividad. Precisamente uno de los elementos que diferencian las plataformas de productos audiovisuales y las cadenas de televisión (por muy temáticas y especializadas que sean) es que exista o no programación de unos contenidos, distribuidos según un orden preciso, en una rejilla de emisión. Sin programación no hay televisión, al menos como se ha entendido desde su nacimiento. 
Aquí se aborda el análisis de los estudios académicos sobre programación televisiva. Por eso el artículo mismo se puede considerar, en cierto sentido, un estado del arte sobre programación televisiva. En realidad va más allá. Porque no se pretende dar cuenta de los modelos académicos de análisis sobre este apartado (la programación televisiva) ni sobre los recursos que los profesionales emplean para tomar decisiones y su justificación. Aquí se analizan las relaciones entre las aportaciones que se han realizado en este campo en el cuerpo de publicaciones que se señalan. Este análisis se plantea además de modo diacrónico en sus apartados: lo que permite mostrar -entre otras cosas- los clústeres de términos y conceptos que conforma la producción científica sobre este campo desde su origen y su evolución. Este aspecto es clave porque los términos que los investigadores emplean para designar ámbitos de interés, y los demás conceptos con que se relacionan (los clústeres) abren una perspectiva que puede orientar sobre el significado de su evolución y el sentido de la investigación académica. Dicho de modo patente: valorar si esta va por delante o detrás de las posibilidades de la programación televisiva en este caso. Indudablemente este análisis solo aporta la vertiente de la investigación académica y sus "preocupaciones" ante la realidad de la programación televisiva y sus efectos.

Y ese análisis se realiza en un doble círculo. El primero, inmediato, se centra en los artículos publicados en la Core Collection de Web of Science (WoS) sobre esta temática. Desde luego, no constituyen la totalidad de las aportaciones académicas sobre el tema, pero es indudable que conforman un corpus enormemente significativo puesto que Web of Science persigue el objetivo de indexar las principales fuentes científicas del mundo (Repiso, Ahedo, \& Montero, 2018). Sobre este set de datos se abordan las cuestiones básicas: artículos y autores más relevantes por sus citas, revistas que más estudios han acogido, etc. Muestran cómo la televisión -la programación en algunos de sus aspectos- constituye un laboratorio de la realidad social para otras áreas de conocimiento, especialmente a las propias de salud y ponen de manifiesto el protagonismo que a este medio se le otorga desde otras disciplinas que se acercan a los estudios sociales.

Es llamativo el enorme auge que han tenido los estudios sobre programación televisiva en un campo ajeno como el de los analistas de la alimentación y de sus 
trastornos, también de los psicológicos. En este sentido, Stice y Shaw (1994) relacionan la presión mediática para alcanzar un cuerpo ideal en las mujeres con estrés, depresión, o síntomas bulímicos. Así mismo, Mulligan, Altmann et al. (2011) sostienen que los medios de comunicación desempeñan claramente un papel importante en la epidemia actual de obesidad infantil y adolescente. Paradójicamente la televisión que se emite se convierte en un remedo de la sociedad en estos estudios y parece confirmar así lo que desde la comunicación se venía sosteniendo desde hace años: el referente de realidad que ofrece la televisión es ella misma, sus emisiones. La programación televisiva es un campo que no sólo afecta a los medios, sino que está presente en otros espacios temáticos. En este sentido podría hablarse, algo más que metafóricamente, de la programación televisiva como de una agenda setting de la vida ordinaria, tan capilar como aparentemente oculta precisamente en su "autonormalidad".

El segundo set de datos de análisis que se aborda de la producción académica es enormemente más amplio: de las aportaciones de la WoS se ha obtenido la literatura científica que cita (esté o no incluida en ella) y con un corpus mucho más amplio se han podido abordar con más garantías (aunque no sea exhaustivo) los análisis de correlaciones de términos en busca de sus correlatos temáticos. Primero, en términos generales sobre el total de la producción incluida; luego, según cortes cronológicos para valorar la evolución y los aspectos diacrónicos del análisis: en el fondo, qué ha ido interesando desde la programación televisiva sobre la televisión y sus implicaciones sociales, culturales, política, temáticas, genéricas, etc.

Del análisis de este segundo corpus se ha podido comprobar que el ciclo de estudios académicos sobre la programación televisiva comienza a descender y abre la posibilidad de que quede reducido al análisis de su historia. No constituiría un inmediato cierre de época (al estilo de los estudios de egiptología faraónica), pero sí un notable descenso que podría acabar con su liquidación práctica en la WoS con lo que ello significaría de tendencia.

La evolución, década a década, de la producción académica sobre programación televisiva ofrece indicadores de tendencia que tiene interés resumir aquí. El primero, ya se ha mencionado: la reducción de los estudios sobre programación te- 
levisiva. Es el correlato de la aparición y consolidación de una nueva televisión: a la carta y de pago, frente a la abierta y generalista clásica que puede considerarse que ha iniciado su extinción (proceso que será largo y que no quita importancia a esta modalidad en la actualidad) y que limitará sus audiencias a las de menor nivel económico (Turner \& Tay, 2009). Habrá que ver cómo resuelve la publicidad esta contradicción y qué resultará para la televisión en abierto. Quizá quedar reducida a la televisión estatal de servicio público. Habrá que ver entonces qué papel juega el entretenimiento en ese servicio público con unas audiencias acostumbradas a un entretenimiento muchas veces de muy bajo nivel.

La otra línea de tendencia que muestra el análisis, seriado cronológicamente, de palabras es la tendencia a considerar la televisión como simple objeto de la investigación en comunicación por su protagonismo creciente en la vida social, política, económica (publicidad) a su papel clave en la definición de estándares en la cultura popular. El reconocimiento general de esta importancia social de la televisión (y de su programación, porque eso es lo que se emite y ven los espectadores) deriva progresivamente a su papel no tan positivo en extensión de algunos males. Primero en los desórdenes alimentarios (Stice, Schupak-Neuberg, et al. 1994) y la disminución de la actividad física en niños y adolescentes inicialmente (Taras, Sallis, et al., 1989; Taras \& Gage, 1995). Luego, en la difusión de estereotipos hirientes para minorías: raza (Mastro \& Greenberg, 2000), inmigración (Mastro \& Behm-Morawitz, 2005) y género (Rozario, Masilamani, et al., 2018). El resultado ha sido la consideración de la programación televisiva como algo socialmente nocivo. Es significativo que la mayor parte de los estudios que se centran en estas cuestiones (aunque no todos) los hayan realizado investigadores de otras áreas: especialmente Medicina, Psicología y Sociología.

\section{Material y métodos}

Este trabajo analiza los resultados de investigación sobre Programación de Televisión desde una perspectiva bibliométrica, aunque procura abrirse a otras consideraciones desde este punto. En concreto, se aplican técnicas de Análisis de Redes Sociales a documentos científicos. Se plantea los siguientes objetivos: 
130 | Alicia Moreno-Delgado, Rafael Repiso y Julio Montero-Díaz MONOGRÁFICO

1. Descubrir en qué áreas de conocimiento se publican los trabajos identificados sobre Programación de Televisión.

2. Identificar los documentos más relevantes para los propios investigadores sobre Programación de Televisión.

3. Identificar los temas predominantes que se han abordado en las investigaciones sobre Programación de Televisión en su recorrido diacrónico.

Es una investigación de carácter fundamentalmente descriptivo en la que se emplean técnicas cuantitativas. Para los dos primeros objetivos, se procedió a identificar los trabajos en la Web of Science Core Collection mediante una primera búsqueda con las siguientes palabras clave: TV programming, Broadcast Schedule, TV guide y Broadcast programming. Se identificaron 345 registros. A continuación, se analizaron estos registros utilizando Excel y VOSviewer. Eso posibilitó realizar una descripción bibliográfica en la que se identificaron: los autores, trabajos y revistas más citados, así como áreas y países de producción. Este trabajo incluye trabajos externos a WoS: se recogieron en una base de datos en formato RIS.

Tras completarse esta primera búsqueda y para alcanzar el tercer objetivo, se procedió a analizar la bibliografía citada por los documentos obtenidos $\mathrm{y}$, a continuación, la bibliografía citada de ese segundo grupo de documentos en una estrategia de "bola de nieve". Cuando por saturación dejaron de aparecer nuevas referencias, se puso fin al proceso. Se identificaron un total de 733 documentos que se han analizado.

Toda la información obtenida se almacenó y organizó utilizando Excel y Mendeley para, a continuación, generar un archivo RIS que se pudiera utilizar por el programa VOSviewer (van Eck \& Waltman, 2010). También se empleó Pajek, un software para analizar redes de co-palabras (Batagelj, 2008). Para poder realizar el análisis de redes sociales de co-palabras en títulos y resúmenes se tradujeron al inglés todos los títulos y resúmenes de documentos que estaban en otros idiomas. Para esta tarea se empleó el programa Deepl. 
Para el análisis de redes se tuvieron en cuenta los términos incluidos en el título, el resumen y las palabras claves de los documentos señalados. Además, se realizó una distribución de trabajos por década que cubrieran toda la cronología abarcada. Se establecieron las etapas siguientes: 1. registros publicados hasta el año 1970 (con 24 documentos); 2. 1970-1980 (con 47 documentos); 3. 1980-1990 (46 documentos); 4. 1990-2000 (97 documentos); 5. 2000-2010 (116 documentos); 6. 2010-2018 (58 documentos). Como puede observarse, se solapan los años terminados en cero entre periodos. Así se da continuidad a los términos empleados en los documentos de estos años en dos periodos. Las redes sociales han sido representadas con el algoritmo de Kamada Kaway (Kamada \& Kawai, 1988) y sus grupos de palabras con el algoritmo de clústeres de Louvain (Blondel, Guillaume, Lambiotte, \& Lefebvre, 2008). Todo lo descrito puede observarse en la Tabla 1 que resume la metodología para la consecución de los objetivos planteados.

\begin{tabular}{|l|l|l|}
\hline \multicolumn{1}{|c|}{ Objetivos } & \multicolumn{1}{|c|}{ Obtención De Datos } & $\begin{array}{l}\text { Herramientas de análisis } \\
\text { de datos }\end{array}$ \\
\hline $\begin{array}{l}\text { Descripción bibliográfica: } \\
\text { Identificación de los trabajos } \\
\text { más citados } \\
\text { Autores más citados } \\
\text { Revistas más citadas } \\
\text { Áreas de producción } \\
\text { Países de producción }\end{array}$ & Web of Science Core Collection & $\begin{array}{l}\text { Excel } \\
\text { VOSviewer }\end{array}$ \\
\hline $\begin{array}{l}\text { Redes sociales } \\
\text { Redes de co-palabras } \\
\text { Distribución temporal }\end{array}$ & & \\
\hline
\end{tabular}

Tabla 1: Metodología empleada en el estudio para la consecución de los objetivos planteados. Fuente: Elaboración propia.

Este estudio utiliza, por tanto, dos grupos de datos. Uno lo forman los 345 documentos para la descripción bibliográfica (WoS Core Collection). Luego, un segundo set de datos de 733 documentos que se emplea para el análisis de redes (WoS Core Collection + documentos citados). Todos los datos se han obtenido entre los meses de octubre y noviembre de 2018. 


\section{Resultados}

El primer objetivo de este trabajo es la descripción bibliográfica de los artículos que abordan la Programación en Televisión. Si se analiza la distribución por áreas de los 345 documentos de la WoS, se observa que la categoría de Comunicación y la de Cine, Radio y Televisión acaparan la mayoría de la producción académica sobre programación en televisión. De hecho, conforman más del 50 por ciento del total (Gráfico 1).

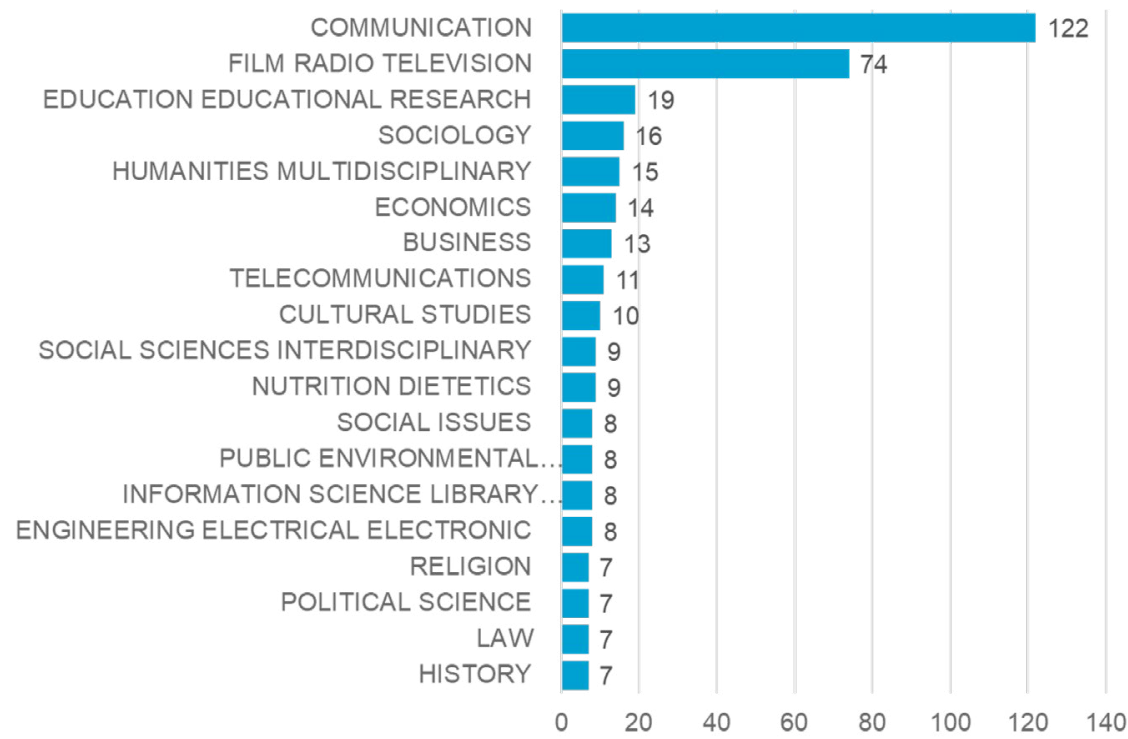

Gráfico 1: Distribución de trabajos por áreas. Hay que tener en cuenta que el total de trabajos por área supera el número total de trabajos ya que hay documentos que pertenecen a más de una categoría de la WoS.

Fuente: Elaboración propia.

En cuanto a las revistas que publican estos trabajos, se han identificado 11 títulos con más de 5 artículos: Journal of Broadcasting \& Electronic Media es la más productiva con 18 documentos publicados. Le sigue Journal of Communication con 10 documento (Gráfico 2). 
Análisis de redes sociales de la producción científica sobre programación televisiva | 133

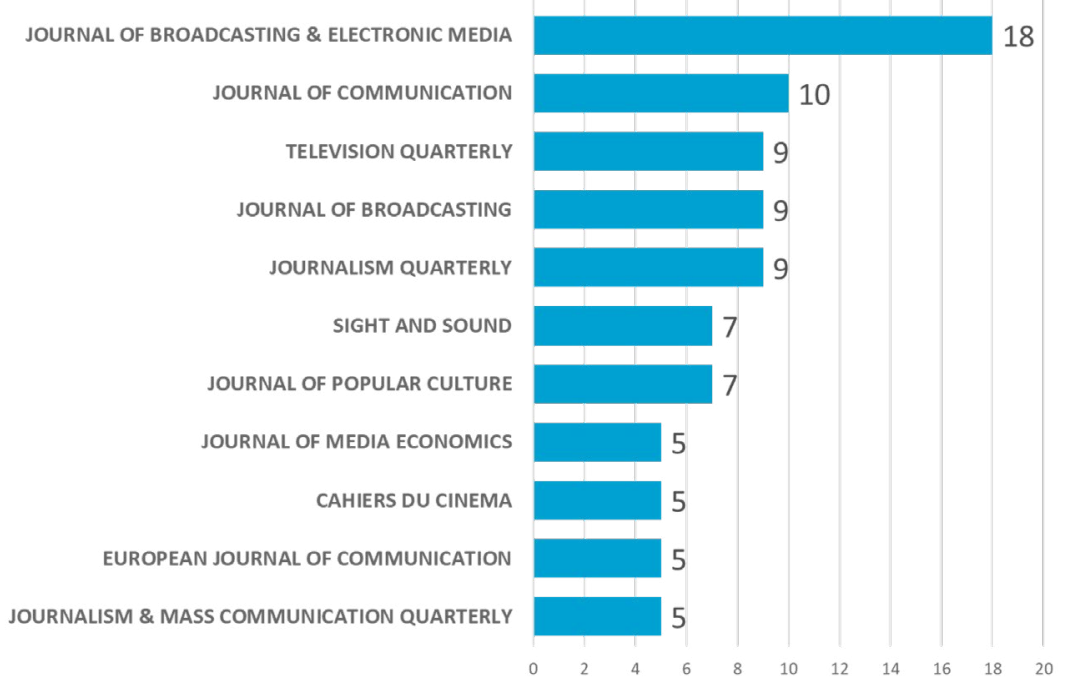

Gráfico 2: Revistas más productivas (más de 5 artículos).

Fuente: Elaboración propia.

Ambas cifras (etiquetas temáticas predominantes y áreas de conocimiento de las revistas que más papers publican) tienen interés. Pero también lo ofrece la amplia diversidad de temas a los que se asocian otras etiquetas temáticas tan lejanas aparentemente de la programación televisiva: Medio Ambiente, Estudios Culturales, Nutrición; Tendencias Sociales; Religión; Ciencia Política; Historia... Y todo ello suscita la cuestión de qué interés pueda tener la programación televisiva en esos campos.

Por otro lado, el análisis de los documentos por países (Gráfico 3) muestra cómo España, con 14 publicaciones, se sitúa en tercer lugar por detrás de EE. UU. con 170 trabajos, y de Canadá que acumula 15 documentos. El sesgo anglosajón de la WoS y la importancia de la televisión en Estados Unidos permite entender estas cifras y ayuda a valorar la posición de la producción en española que se sitúa a un nivel similar al de Gran Bretaña y Canadá. 
134 | Alicia Moreno-Delgado, Rafael Repiso y Julio Montero-Díaz

MONOGRÁFICO

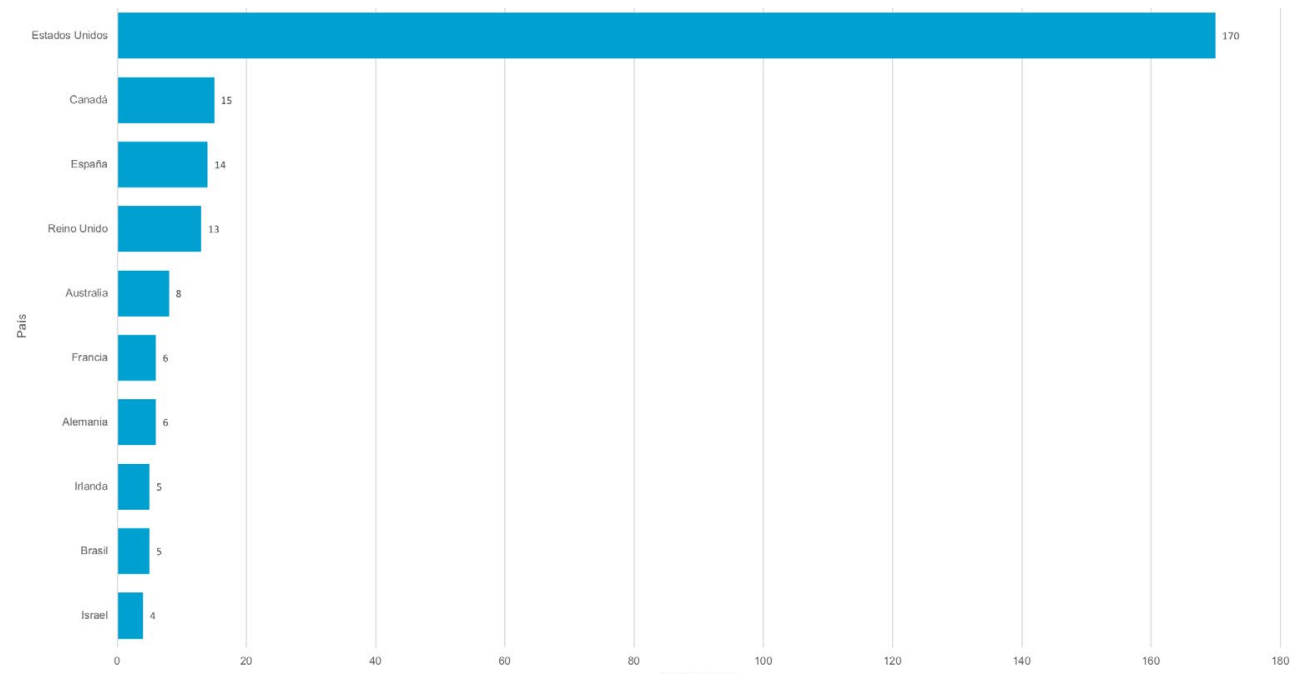

Gráfico 3: Distribución de trabajos por paises.

Fuente: Elaboración propia.

Al analizar los documentos encontrados sobre programación de Televisión por década, la distribución de los trabajos, tal y como se observa en el Gráfico 4, es la siguiente: una tendencia ascendente hasta la década de 2000-2010 y en descenso en los últimos años en cuanto a producción científica.

Documentos por décadas

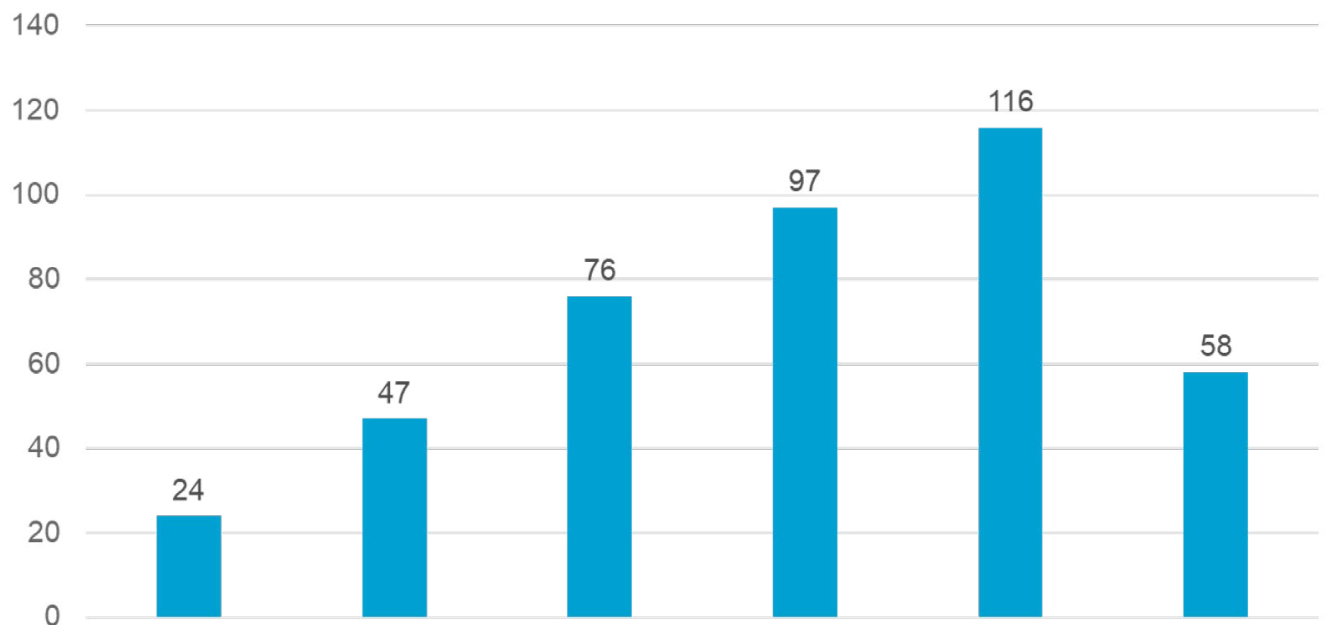

Gráfico 4: Evolución de la producción científica por décadas.

Fuente: Elaboración propia. 
La identificación de los autores y documentos más relevantes entre los propios investigadores (segundo objetivo de este trabajo) se ha realizado mediante el análisis de redes sobre los autores co-citados. Es decir, cómo se citan entre ellos en los trabajos sobre Programación de Televisión. Hay seis grupos, alrededor de seis autores, que destacan notablemente sobre el resto (Gráfico 5). Es importante señalar que, de estos seis autores, solo dos pertenecen al área de Comunicación. Los otros cuatro son de Psicología y Medicina. Los autores de cabecera que más referencias agrupan son Thomas N. Robinson, que puede identificarse en el clúster rojo, con 81 citas; Eric Stice (clúster azul) y Albert Bandura (clúster amarillo) con 114 citas; M. E. Goldberg (clúster turquesa) con 40 citas; Dale Kunkel (clúster morado) citado 62 veces; y Annie Lang (clúster verde) cuyos trabajos sobre este ámbito han recibido un total de 58 citas.

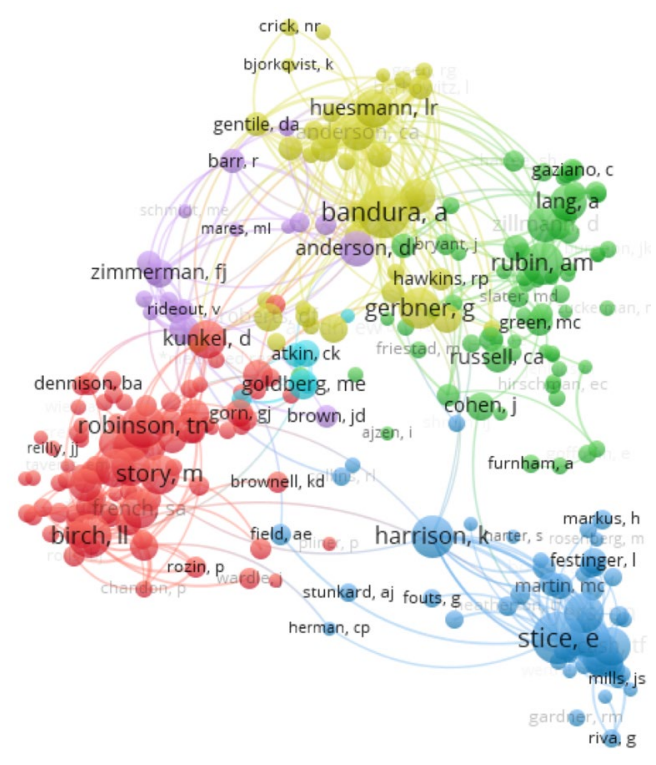

Gráfico 5: Red de autores co-citados.

Fuente: Elaboración propia.

En cuanto a los trabajos más citados (Tabla 2), la primera posición la ocupa The elastic body image: The effect of television advertising and programming on body image distortions in young women. Se publicó en una revista del área de Comunicación. Sin embargo, el segundo y tercer documento con más citas son del ámbito de la Medicina. 


\begin{tabular}{|l|c|}
\hline \multicolumn{1}{|c|}{ Trabajos } & $\begin{array}{c}\text { Número } \\
\text { de citas }\end{array}$ \\
\hline $\begin{array}{l}\text { Myers Jr, P. N., \& Biocca, F. A. (1992). The elastic body image: The effect of tele- } \\
\text { vision advertising and programming on body image distortions in young women. } \\
\text { Journal of communication, 42(3), 108-133. }\end{array}$ & 66 \\
\hline $\begin{array}{l}\text { Kotz, K., \& Story, M. (1994). Food advertisements during children's Saturday morn- } \\
\text { ing television programming: are they consistent with dietary recommendations? } \\
\text { Journal of the American Dietetic Association, 94(11), 1296-1300. }\end{array}$ & 63 \\
\hline $\begin{array}{l}\text { Taras, H. L., Sallis, J. F., Patterson, T. L., Nader, P. R., \& Nelson, J. A. (1989). Tele- } \\
\text { vision's influence on children's diet and physical activity. Journal of Developmental } \\
\text { and Behavioral Pediatrics. }\end{array}$ & 30 \\
\hline
\end{tabular}

Tabla 2: Artículos más citados de Programación en Televisión en las investigaciones sobre dicho tema. Fuente: Elaboración propia.

Desde el punto de vista bibliométrico esas cifras manifiestan la mayor capacidad de citas que generan las áreas de Salud frente a las Ciencias Sociales en general y de la Comunicación en particular.

Al realizar el análisis de redes de co-palabras para determinar en qué aspectos se centran las investigaciones realizadas sobre Programación de Televisión a partir del título y resumen de los documentos, encontramos 9 grupos diferenciados (Gráfico $6)$, que señalan una clusterización clara.

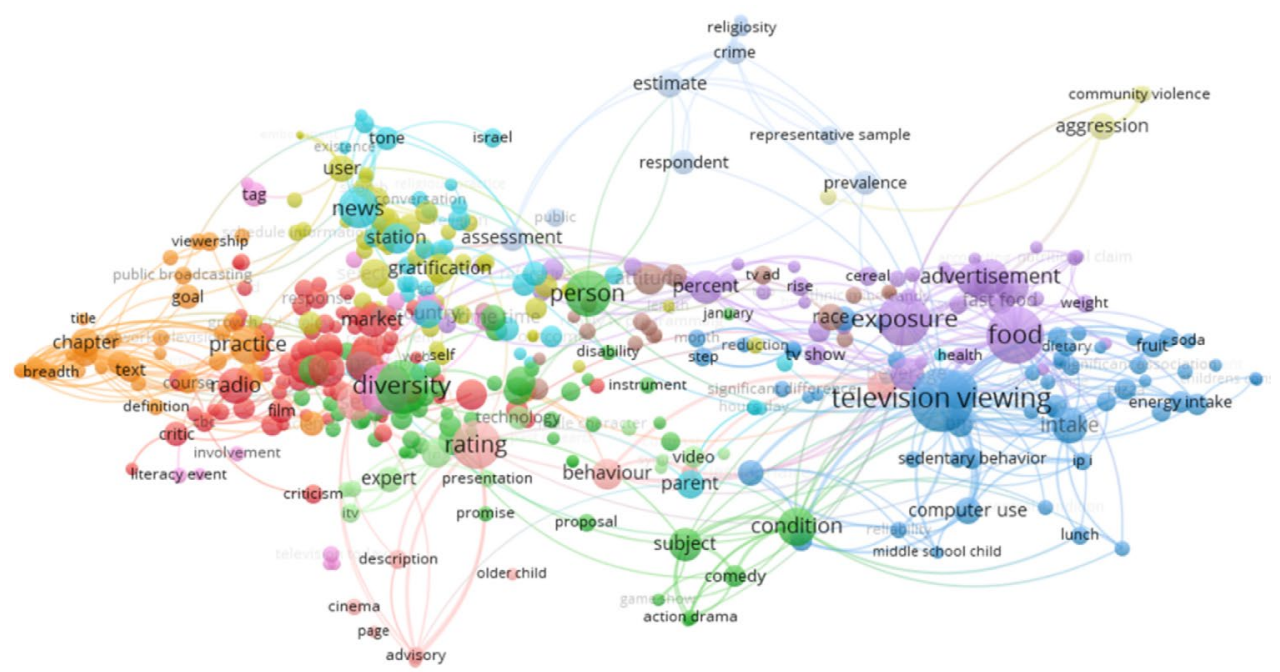

Gráfico 6: Red de co-palabras para documentos sobre Programación de Televisión. Fuente: Elaboración propia. 
En el clúster de color rojo los términos History y Competition son los más relevantes. Delimita un bloque centrado predominantemente en los aspectos históricos de la programación en el que la competencia centra una buena parte de la línea temática en la investigación sobre programación televisiva. En el clúster verde destacan Diversity y Person. Es también claro: diversidad y consiguientemente sobre el término persona, que es una de las maneras de referirse a ese ser distinto y que tanto interés suele despertar para los programadores el conjunto de términos. En color azul Television viewing es el concepto más destacado. El ver televisión no ofrece dudas en relación con los estudios de programación. En el clúster amarillo aparecen Gratification, Channel repertoire, User y Consumer como palabras más relevantes. Los términos que relacionan uso y consumo, junto con repertorio de programas de cada canal, conforman también un grupo de términos que ayudan a entender que ese tipo de trabajos estén relacionados entre sí. En el conjunto identificado con el color morado Food, Exposure y Advertisement son las palabras más utilizadas. Este grupo de términos reunidos en torno a publicidad, alimentos y exposición ante la programación tiene también consistencia: manifiesta de hecho una de las preocupaciones dominantes sobre los efectos (negativos) de la televisión. News, Station, Tone y Country son las más utilizadas del clúster turquesa. Hay también lógica en las relaciones internas que manifiestan estos términos: la información televisiva está muy relacionada en Estados Unidos (el país que agrupa más de la mitad de las publicaciones analizadas) con las estaciones locales de emisión, lo que conforma igualmente relaciones patentes con el tono y el territorio. Las relaciones más representativas del clúster naranja son Practice, Chapter y Collection. Si antes eran las noticias ahora es la ficción televisiva la que engarza los términos más citados. En el grupo de color marrón destacan Attitude, Race y Agression. Volvemos a algunos de los efectos de la programación de televisión en el ámbito social: actitud, raza y agresión, sitúan en discriminación. Finalmente, el clúster donde el término más relevante es TV Guide nos habla de una de las fuentes más frecuentemente utilizadas por los académicos en sus investigaciones.

El tercer objetivo es identificar qué temas se han tratado en las investigaciones sobre Programación en Televisión a lo largo de la historia. El análisis de redes de co-palabras por década refleja tanto los términos más utilizados como las relaciones entre los mismos. Lo primero es el incremento constante del número de documentos (hasta la década que se inicia en 2010 y aún no está cerrada) y también de intensidad y complejidad de las 
redes de citación. Se muestran también, a partir de las redes de co-palabras, los términos que aparecen únicamente en una determinada década o que se emplearon en títulos o palabras claves de forma muy destacada sobre el resto de los periodos analizados.

Hasta 1970 (Gráfico 7) se aprecia una red con un bajo número de relaciones. Inferior al de décadas posteriores. Se han detectado 5 grupos en los que se observan las diferentes temáticas de los años analizados. Así, en el clúster rojo destacan palabras como television program type o audience; en el grupo de términos en color azul destacan effect e interest; en el clúster verde donde las bolas que representan las palabras tienen un tamaño similar, se identifican términos como violence, danger o respect entre otros; en el grupo rosa, el más pequeño de todos, destacan medium y model; $\mathrm{y}$, finalmente, en el clúster amarillo programming, area o university.

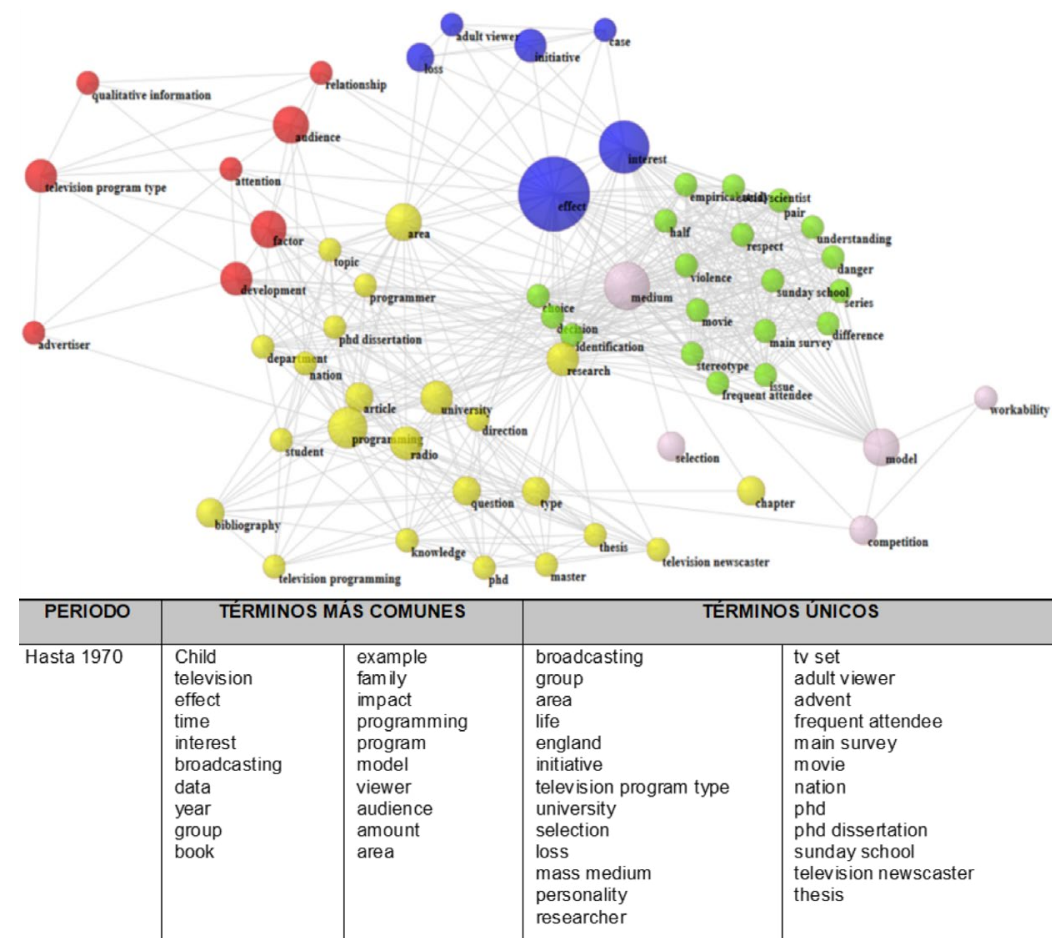

Gráfico 7: Red de co-palabras a partir de documentos publicados antes del año 1970 y relación de términos presentes en la red.

Fuente: Elaboración propia. 
Análisis de redes sociales de la producción científica sobre programación televisiva | 139

En la década de los setenta se aprecia ese aumento de relaciones y de términos y se diferencian 4 grupos con palabras destacadas (Gráfico 8). Grupo rojo: model, show. Grupo Azul, el más pequeño compuesto por siete conceptos: comedy, condition. Grupo verde, con un número de términos superior, pero muchos de ellos con escasas conexiones: broadcasting, station, economic. Grupo amarillo: $C B C$, paper, radio.

La red es aún tenue y la intensidad está relacionada con los términos más generales: Medium, Model, Interest, Effect. Las relaciones entre los demás se diluye en comparación con estas.

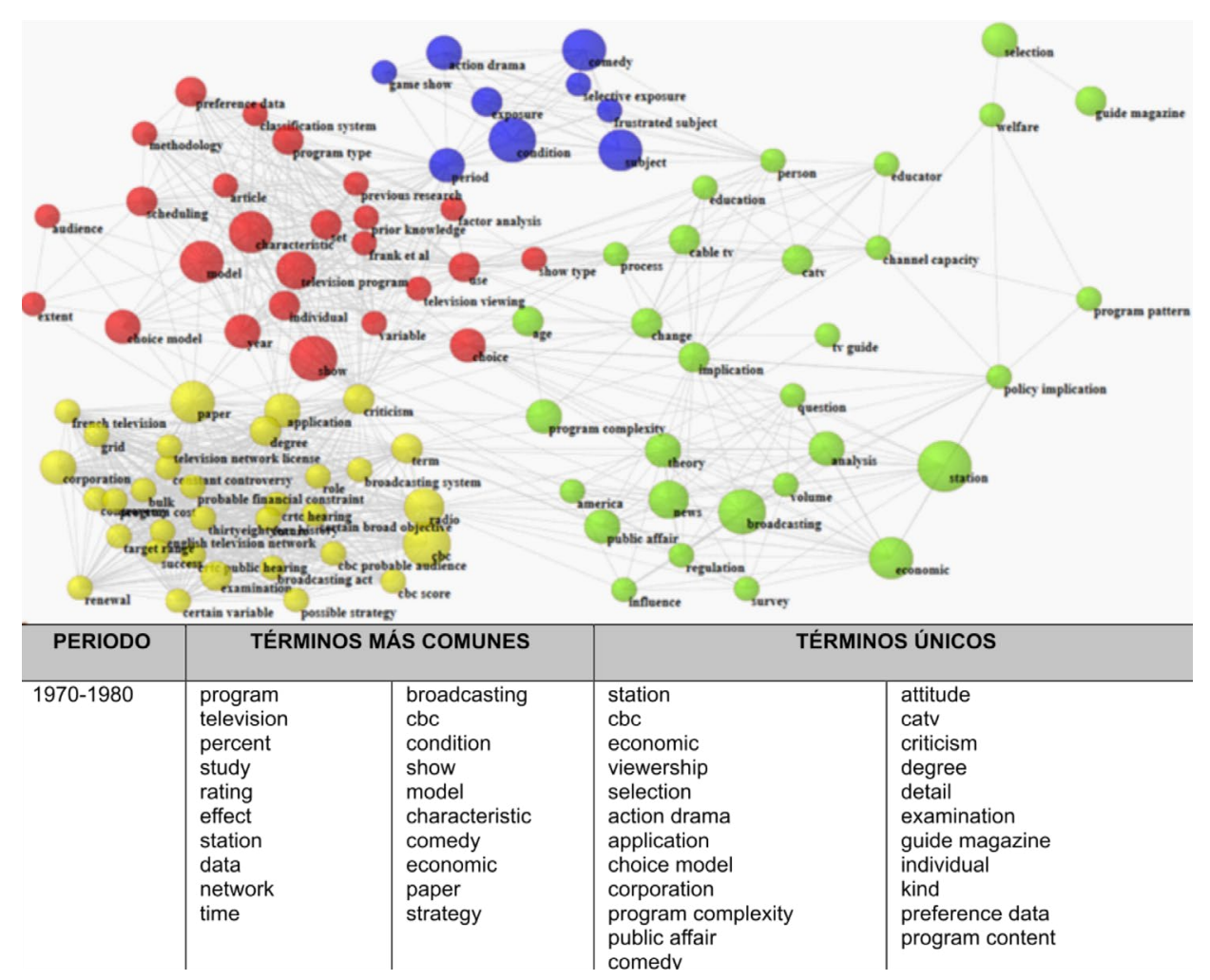

Gráfico 8: Red de co-palabras a partir de documentos publicados entre 1970 y 1980 y relación de términos presentes en la red.

Fuente: Elaboración propia. 
140 | Alicia Moreno-Delgado, Rafael Repiso y Julio Montero-Díaz MONOGRÁFICO

En los años comprendidos entre 1980 y 1990 se observa muy claramente un aumento de conceptos y la red se vuelve más compleja (Gráfico 9), lo que se traduce en un mayor número de clústeres. Se han considerado 12 grupos. El menor de ellos lo conforman 3 palabras (en color gris) frente a las 26 que forman el de mayor tamaño (en color amarillo). Del conjunto de palabras, destacan pattern, child, programme, time, behavior y tv guide.

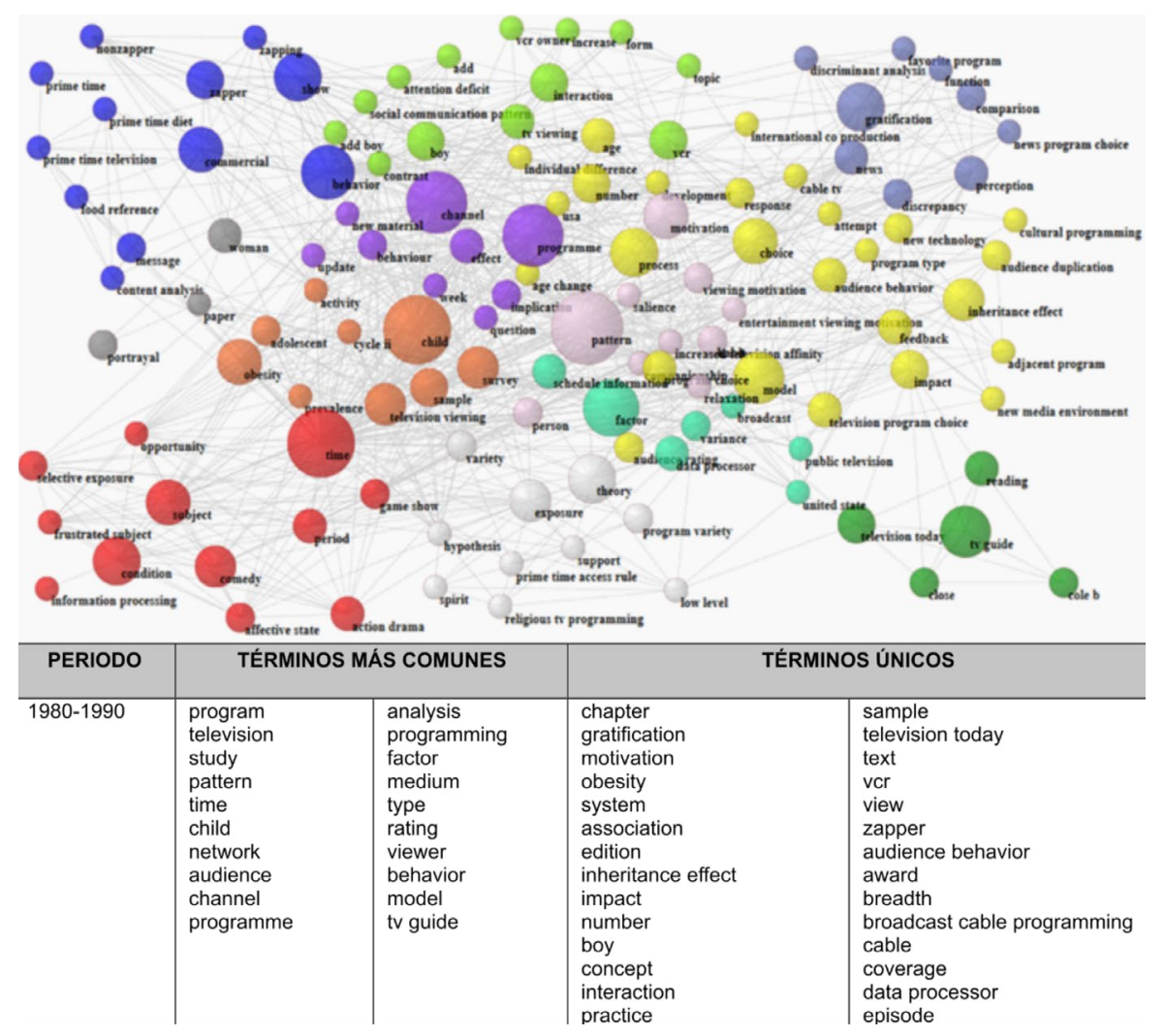

Gráfico 9: Red de co-palabras a partir de documentos publicados entre 1980 y 1990 y relación de términos presentes en la red.

Fuente: Elaboración propia.

El análisis de la red de co-palabras entre los años 1990 y 2000 (Gráfico 10), muestra 7 grupos diferenciados. Las relaciones entre los términos aumentan y entre ellos destacan television de forma significativa sobre el resto. A continuación, aparecen study y diversity, child y program. 
Análisis de redes sociales de la producción científica sobre programación televisiva | 141

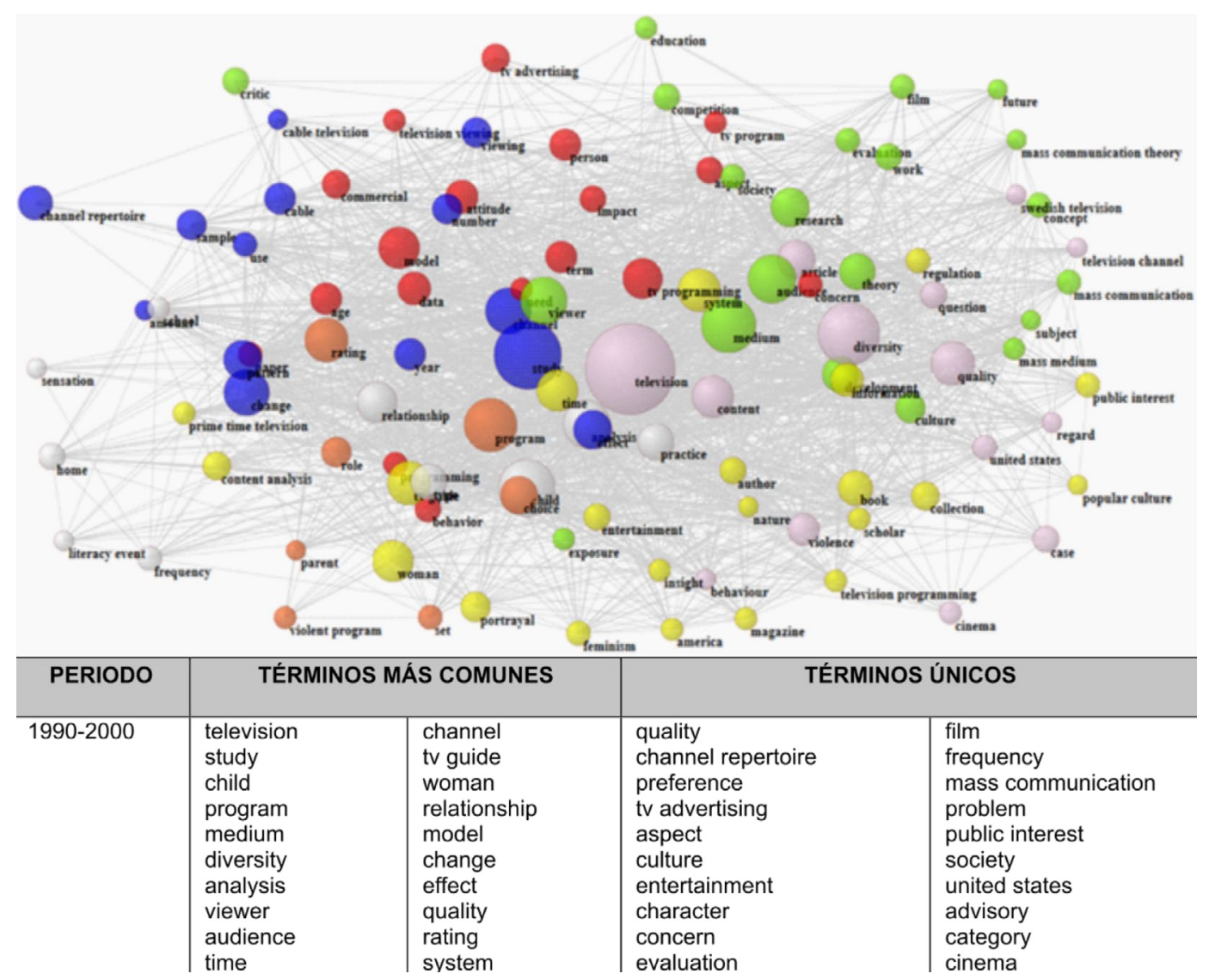

Gráfico 10: Red de co-palabras a partir de documentos publicados entre 1990 y 2000 y relación de términos presentes en la red.

Fuente: Elaboración propia.

La última década completa estudiada, 2000-2010, es la que cuenta con mayor número de trabajos sobre Programación de Televisión. Esto se refleja en el análisis de redes donde las relaciones son más complejas que en el resto de los periodos: mayor número de documentos, mayor número de palabras, mayor número de conexiones (Gráfico 11). En esta red, formada por 5 grupos, los términos más destacados: television, child, study, television viewing, content y food. 


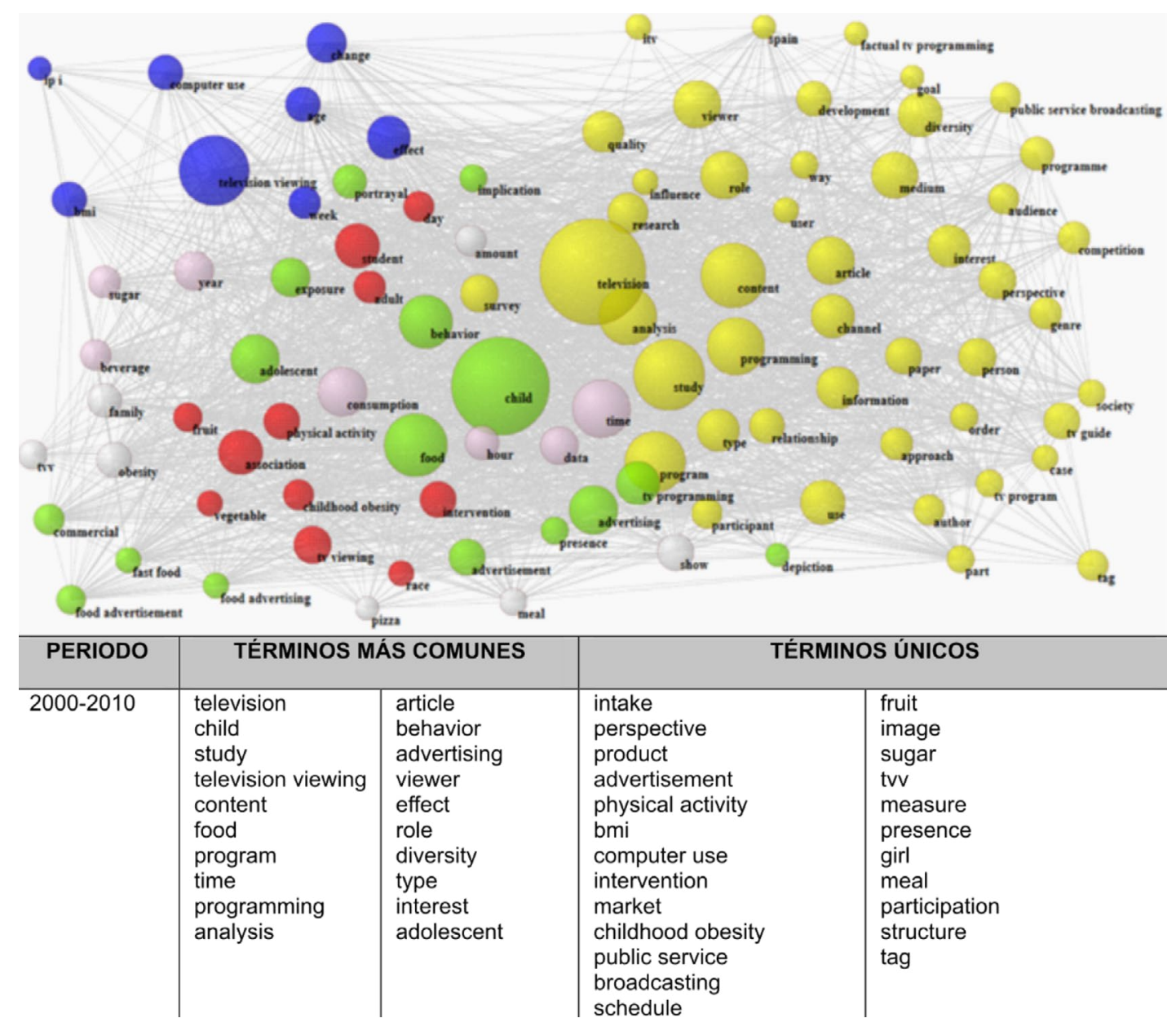

Gráfico 11: Red de co-palabras a partir de documentos publicados entre 2000 y 2010 y relación de términos presentes en la red.

Fuente: Elaboración propia.

Finalmente, entre el año 2010 y el 2018, por primera vez hay menos documentos que en la década anterior (Gráfico 12). Aunque es cierto que los años incluidos son menos, es patente que la tendencia es descendente. 
Análisis de redes sociales de la producción científica sobre programación televisiva | 143
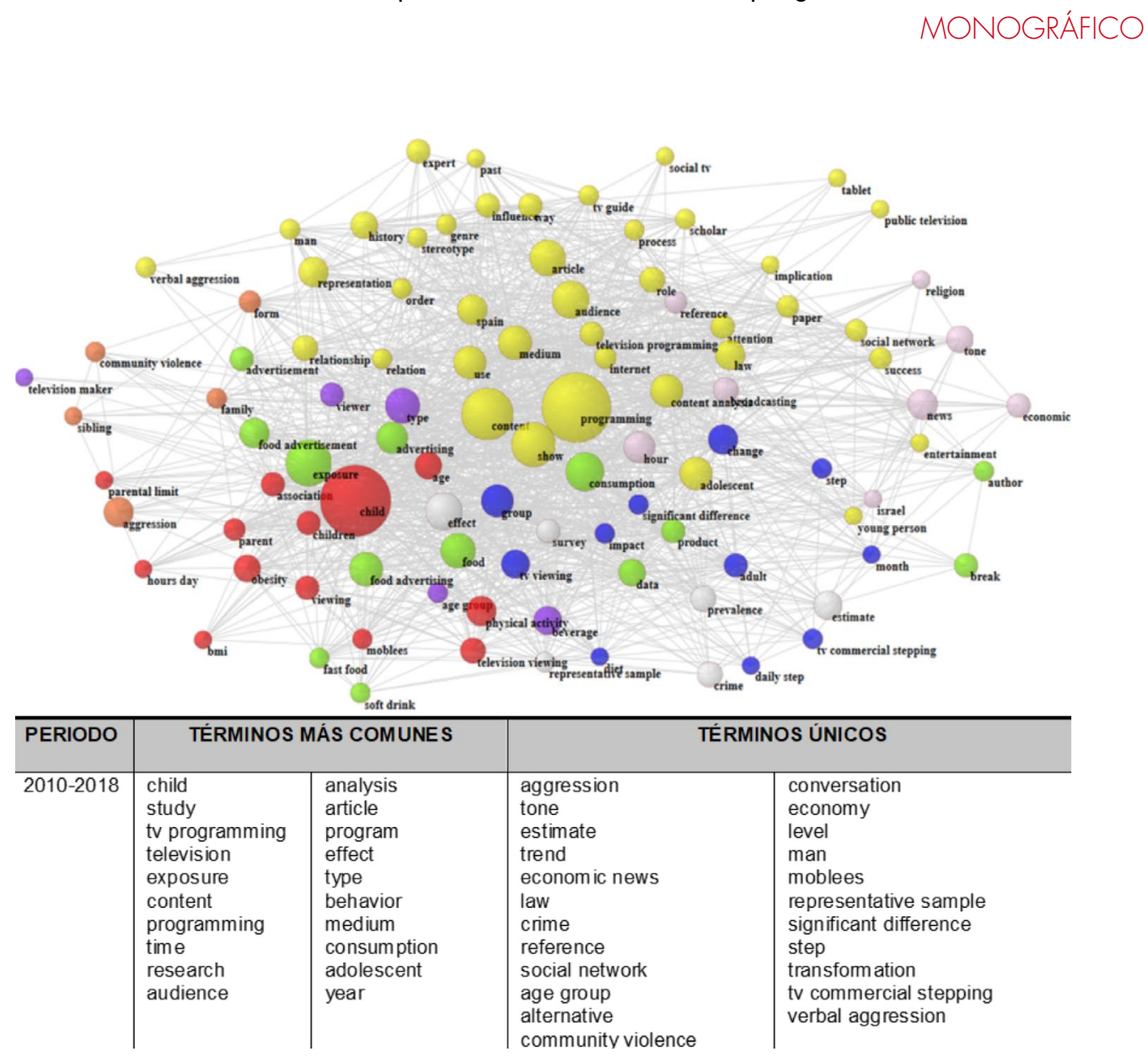

Gráfico 12: Red de co-palabras a partir de documentos publicados entre 2010 y 2018 y relación de términos presentes en la red.

Fuente: Elaboración propia

En términos generales esta baja relativa de protagonismo del medio se traduce en un descenso de la red de co-palabras generada, que además vuelve a simplificarse. Se aprecian 8 grupos donde se repiten los términos relevantes identificados en los años del 2000 al 2010: Child, Study, Television.

\section{Conclusiones y Discusión}

Después de analizar la producción científica sobre programación televisiva, el presente estudio ha llegado a las siguientes conclusiones: 
En la descripción desde una perspectiva bibliométrica se han identificado 19 campos de investigación (con un mínimo de 7 registros) en los que se publican trabajos sobre Programación de Televisión. De ellos, las categoría de Communication y Film Radio Television agrupan a más del 50 por ciento del total de los registros, por lo que son los más relevantes en lo que se refiere a publicaciones sobre programación televisiva (Gráfico 1). Hay coherencia. La programación televisiva se aborda desde el área de Comunicación en primer lugar y de manera destacada. También es lógico que se ocupen de ella desde el área de Cine, Radio y Televisión. Tiene también su lógica que la televisión interese desde la Educación, Sociología, Humanidades, etc. La importancia de la programación televisiva (tan vinculada a sus emisiones y a lo que de la televisión es visualmente más patente) se destaca especialmente.

La misma lógica de normalidad se sigue del análisis de publicaciones que más aportaciones sobre programación televisiva recogen: primero una de Televisión y en segundo lugar otra de Comunicación (Gráfico 2). Las siguientes posiciones con cifras significativas siguen la misma tendencia: una cierta superioridad de las que dedicadas a Televisión y a las de Comunicación. Luego aparecen otras de carácter más circunstancial, pero siempre dentro de los estudios de Imagen o de Comunicación.

En cuanto a los trabajos más destacados, presentados en la Tabla 2, se han identificado los siguientes: Myers \& Biocca (1992) citado en los trabajos seleccionados 66 veces; Kotz y Story (1994) con 63 citas; y Taras, Sallis, et al. (1989) que ha recibido 30 citas. El primer documento se encuentra publicado en una revista del área de Comunicación mientras que los otros dos registros se integran dentro del área de la Medicina.

Es de interés una consideración paralela: estos estudios realizados por especialistas de áreas ajenas a los estudios sobre programación televisiva se acercan a ella por su protagonismo en la vida social y cultural de sus países. En concreto sobre los efectos de esta (de la programación televisiva) sobre los hábitos alimentarios. Constituye, por lo tanto, un reconocimiento de su importancia. Además, en este tipo de trabajos late una concepción de la televisión como laboratorio social, que, 
en el fondo, reconoce una conexión medio-realidad social no tan clara entre los estudiosos de la Comunicación. Es verdad que esta identificación de fondo entre la realidad representada y la realidad se limita a campos concretos y no se universaliza, pero tiene interés destacarlo.

Además, el análisis de redes de los autores co-citados, es decir, cómo se citan entre ellos los autores del ámbito, ha permitido detectar los autores de cabecera (Gráfico 5). En este sentido, Thomas N. Robinson, con 81 citas; el Eric Stice y Albert Bandura con 114 citas cada uno; M. E. Goldberg con 40 citas; Dale Kunkel citado 62 veces; y Annie Lang con un total de 58 citas, son los más relevantes entre los investigadores sobre Programación de Televisión. Es importante destacar que los autores que no son de las áreas de Comunicación o Cine, Radio y Televisión, reciben sus citas de colegas de sus áreas respectivas donde el volumen de publicaciones es notablemente mayor que en las citadas. Esto significa que, aunque obtengan más citas, su influencia no es grande entre los estudiosos de la programación televisiva y por lo tanto la superioridad bibliométrica no se traduce en un reconocimiento de autoridad intelectual entre los que cultivan el análisis de la programación televisiva desde las áreas de Comunicación o Cine, Radio y Televisión. Este dato confirma lo que se señala arriba, porque estas áreas reúnen muchísimas más revistas que las paralelas de Comunicación y Cine, Radio y Televisión. No es extraño por eso que se sitúen en las primeras posiciones.

Por otro lado, ante la pregunta de qué países proceden las investigaciones publicadas, la mayoría de ellas, 170 trabajos, se han elaborado en Estados Unidos. España ocupa la tercera posición muy alejada del dato anterior con 14 publicaciones, y por detrás de Canadá que acumula 15 investigaciones (Gráfico 3). En realidad, puede hablarse de una producción abrumadoramente superior norteamericana y de un grupo de tres países (Canadá, España y Reino Unidos) con cifras muy similares y a una enorme distancia, pero también es reflejo de dos fenómenos y es la importancia que en estos países ha tenido la televisión, su democratización, sus estudios científicos sobre la misma y algo que es también importante, la naturaleza anglosajona del producto utilizado, Web of Science. 
Sí que tiene interés resaltar que la producción científica española en este ámbito es significativa y mayor que la de países como Francia y Alemania. Estos datos tienen un significado más claro si se considera que la producción académica publicada en WoS suele considerarse la de mayor relieve y producción internacional.

Al analizar los documentos sobre programación de Televisión por década, la distribución de los trabajos, tal y como se observa en el Gráfico 4, muestra una tendencia ascendente hasta la década de 2000-2010. Desde entonces desciende la producción. La pregunta obligada es si los estudios de televisión han comenzado a pasar a un segundo plano en el interés de los académicos por su análisis. El descenso de estudios académicos sobre televisión en la última década (en este caso se aborda específicamente la programación televisiva que recoge un elemento esencial de la televisión clásica, no de las ofertas a la carta) puede ponerse en relación con dos fenómenos al menos. El primero, que el estudio de internet y de las redes sociales por lo que tiene de innovadores constituye un campo más atractivo para las nuevas generaciones de investigadores. Esto se relacionaría con el que las investigaciones sobre redes sociales atraen más a los investigadores más jóvenes que pueden prescindir de los estudios previos y del background que exigen las investigaciones sobre televisión. A esto se sumaría su mejor preparación para los análisis cuantitativos y la minería de datos que exigen los análisis de redes sociales e internet (Hayama, 2018). Eso sin contar con que la propia televisión pueda considerarse integrada en la web, y no tanto como un medio independiente (Bondad-Brown, Rice, \& Pearce, 2012).

Por otro lado, el estudio de redes de co-palabras ha permitido identificar los temas tratados en las investigaciones sobre Programación en Televisión a lo largo de la historia en lo que parece un ciclo completo que comienza a cerrarse. En este sentido, se han diferenciado dos grandes bloques de estudios de programación televisiva. En primer lugar, se ha identificado un área general que se dedica a estudiar la programación televisiva de forma directa. Se abordan allí los siguientes aspectos: Evolución (Wakshlag \& Adams, 1985); Historia (Dow, 1996); Competencia (Savage \& Wirth, 2005); Contenidos sobre humor, noticias (Buckalew, 1969), series, películas o programas; y Publicidad (Gabszewicz, Laussel et al., 2004). En segundo lugar, se ha identificado otro que podría denominarse alternativo. En este se 
Análisis de redes sociales de la producción científica sobre programación televisiva | 147

estudian la influencia, casi siempre negativa, de la programación de televisiva en las siguientes dimensiones: Obesidad, principalmente infantil (Landhuis, Poulton et al., 2008); Violencia (Wilson et al., 2002); Racismo (Orbe, 2008); y Estereotipos (Espinar Ruiz, 2006).

En este sentido, el Gráfico 6 muestra cómo en el clúster de color rojo los términos History y Competition son los más relevantes. Constituye una referencia a una cierta primacía de los estudios sobre historia de la televisión o a su contextualización diacrónica. En el segundo, el clúster verde, destacan Diversity y Person, que probablemente haya que referir a la diversidad de efectos del medio. En el tercero, en color azul, Television viewing es el concepto más destacado, el hecho de ver televisión, su análisis constituye otro de los grandes temas académicos sobre la televisión. En el clúster amarillo, el cuarto, aparecen Gratification, Channel repertoire, User y Consumer como como palabras más relevantes. El consumo de televisión y sus motivos y consecuencias se muestran como la temática general que subyace. En el quinto conjunto, identificado con el color morado, Food, Exposure y Advertisement son las palabras más utilizadas. Orientan hacia la publicidad y sus efectos, incluido un campo que se ha atendido especialmente desde áreas médicas y psicológicas: sus afectos sobre la alimentación humana y sus problemas. En sexto lugar, el clúster de azul turquesa tiene como centros los términos: News, Station, Tone y Country. Las noticias: un elemento esencial de la programación televisiva y su relación con la particular organización de cadenas, estaciones y territorios en Estados Unidos. En el clúster naranja, el octavo, son Practice, Chapter y Collection las palabras dominantes. En el noveno; el de color marrón, están Attitude, Race y Agression. Se refieren a estudios centrados en la relación de la televisión con el problema racial y sus efectos.

Finalmente, el análisis de redes de co-palabras por década, ha permitido identificar tanto los términos y las relaciones más repetidas y como las que son exclusivas de cada periodo o, aparecen de forma muy significativa en un periodo determinado y de forma anecdótica en el resto. Su interés se centra en cómo se relacionan los conceptos y sobre qué temas se han interesado más los investigadores, bien de modo más continuado (a lo largo de varios periodos), bien de modo específico y concreto para cada década. Desde luego cada uno de ellos constituye 
solo una orientación; pero este primer paso debería facilitar el seguimiento de temas en la cada vez más necesaria historia de la programación televisiva y, de modo secundario, de la propia historia de la televisión y de su relación con la sociedad. El paralelismo o contraste entre lo que los académicos consideraron fundamental en cada periodo y lo que la historia de la televisión, o la prensa especializada de cada momento, o los informes de cada entonces, tiene interés también para valorar hasta qué punto la investigación de más alto nivel supo acertar con los temas realmente claves.

Por lo que se refiere a las relaciones entre los estudios en cada época, el análisis de las redes da cuenta también de la conformación progresiva de un cuerpo de especialistas en la investigación de la programación televisiva en primer lugar $\mathrm{y}$, de modo, secundario, de los estudios de televisión en general. Permite también atender al establecimiento de clústeres de citación que de modo indirecto dan cuenta de la centralidad de algunos autores e incluso podría rastrearse la existencia de "escuelas" de investigadores. Los datos que aquí se ofrecen facilitarán que pueda darse ese segundo paso en el análisis de la producción científica en este campo.

En los registros hasta el año 1970 (Gráfico7), las referencias a lo universitario, el ámbito en que se desarrollaron las primeras investigaciones sobre lo que podía considerarse en los inicios un nuevo medio, se concretan en el uso de términos tan exclusivamente académicos como thesis, phd y phd dissertation, que desaparecen en los periodos cronológicos posteriores.

De los términos exclusivos del periodo 1970-80 (Gráfico 8), destacan algunos que se refieren muy directamente a aspectos de la programación televisiva y a su análisis académico en general: selection, choice model, program complexity, examination, guide magazine, preference data y program content. Otros tienen interés porque se refieren a programas, géneros o formatos de televisión que no llegaron a cuajar, al menos en su denominación. Constituyen una etapa en un trabajo propiamente académico: dar nombre a las nuevas realidades que el medio ofrecía poniéndolo en antena.

Es muy amplio el número de nuevos términos o de exclusivos del periodo 19801990 (Gráfico 9). De hecho, coincide con la década en que se incrementan de modo 
Análisis de redes sociales de la producción científica sobre programación televisiva 149

muy significativo las publicaciones respecto al anterior (se pasa de 47 a 76, un $40 \%$ de crecimiento). Para entonces también ha crecido el número de términos que se van consolidando. En general son indicadores de una progresiva madurez del campo temático que se analiza en lo que a la investigación académica se refiere.

En los años 1990-2000 (Gráfico 10), el conjunto de términos más utilizados y comunes con otros periodos muestra que el análisis de programaciones televisivas tiene cada vez más en cuenta a los espectadores frente a las programaciones mismas: Child, Audience, Type, Rating, Viewer, etc. Otro grupo de estos términos consolidados anuncian las unidades de análisis: Program, network, Channel, Medium y aún se mantiene Programming. Otras hacen referencia a instrumentos de análisis, bien teóricos (Model), bien de fuentes de información (TV Guide).

La intensidad de las relaciones en el periodo 2000-2010 (Gráfico 11) son un indicador de que el campo de investigación de la programación de televisión está consolidado dentro de los estudios de televisión. Por vez primera aparece entre los términos más repetidos el de Article, que luego volverá a estar presente. Es un indicador indirecto de esta consolidación: la referencia al cuerpo anterior de investigaciones que se suele concretar en artículos de revista académica. Luego se hará referencia a que también, de modo secundario, ofrece pista de interés sobre la consolidación del área científica de los estudios sobre televisión. Este asunto no es extraño, porque en el ámbito del mundo televisivo esta década podría considerarse la etapa cumbre, la edad de oro de la televisión generalista y, por tanto, del protagonismo de la programación en la lucha por las audiencias.

Por lo que se refiere a las palabras consolidadas son válidas en términos generales las consideraciones apuntadas para el periodo anterior. Si se atiende además a las más frecuentes, los receptores potenciales mantienen su protagonismo: Child, televisión Viewing, Adolescent. En este caso los nuevos términos que se introducen en los estudios sobre programación televisiva apuntan en la misma línea. Si esto se hace coincidir con la frecuencia de problemas (Phisical Activity, Childhouse Obesity, Sugar, Meal); la importancia de la publicidad (Product, Advertisement, Market) y los que hacen referencia a la intervención para evitar males sociales (Public Service, Intervention), se dibuja un panorama que señala el reconocimiento probado 
de la influencia de la televisión (de su programación emitida) en las sociedades occidentales.

Se apuntan, normalmente en estudios ajenos al área de Comunicación, pero también desde esta, los peligros del visionado incontrolado de televisión por parte de grupos con menor capacidad de resistencia (niños y adolescentes) y los importantes problemas sociales que esto acarrea. La televisión parece pasar ante los ojos escrutadores de la academia de una forma de entretenimiento barata y al alcance de todos, democrática, la mejor manifestación de la cultura popular, a un potencial (y real) peligro social.

Para los años 2010-2018 (Gráfico 12), la primera consideración ha de hacerse a este nivel: el interés académico por los estudios de programación televisiva se ha reducido, el menos en términos de producción de primer nivel. Este hecho es más sensible en los estudios de televisión porque entre las revistas que recogen la mayor parte de la producción solo hay una (Television Quarterly) dedicada exclusivamente a este medio. En efecto, las que más han atendido a este tema son de comunicación en general (Journal of Communication) o bien abiertas a otros medios (por ejemplo, Journal of Broadcasting and Electronic Media). Las demás tienen temáticas más amplias en las que cupo la televisión mientras este medio fue dominante (por ejemplo, las de Cultural Studies). Por lo tanto, la importancia de la televisión como medio paradójicamente ha desbordado los cauces habituales de publicación científica (las revistas específicas abundantes) y eso la ha hecho más sensible también a la baja de protagonismo social relativo del medio analizado ante otros nuevos en auge (en este caso las redes sociales e internet), aunque siga siendo muy importante.

Un segundo factor debería ponerse en relación con el auge de otros medios que además requieren metodologías de análisis específicas (especialmente cuantitativas y de minería de datos). Las nuevas generaciones de investigadores de la Comunicación tienen más facilidades para centrarse en medios emergentes y de mayor protagonismo previsible en el futuro que en el análisis de la programación televisiva que ha quedado como de interés únicamente para el sector más tradicional y con menos futuro de la televisión: la generalista en abierto. Las nuevas formas de 
televisión apuntan a contenidos a la carta (donde la programación carece casi de importancia) y de pago (lo que reduce su impacto social amplio).

Más relevancia tiene otro factor: la consideración negativa de la televisión. Si en la década anterior se produjeron trabajos sobre la mala influencia de la programación televisiva en los grupos sociales más débiles por edad (niños y adolescentes), los nuevos términos que aparecen intensifican aún más esta negatividad: Agression, Crime, Community Violence, Verbal Agression.

Otras conclusiones se refieren al auge y caída de la importancia de los estudios académicos sobre programación televisiva en dos dimensiones: la propiamente académica por el descenso de la producción en revistas WoS durante el último periodo y la que ha ocasionado la evolución del propio medio televisivo, de la televisión generalista en abierto a la oferta diferenciada a la carta y de pago, y su importancia decreciente ante el protagonismo de redes sociales e internet.

\section{Agradecimientos}

Esta investigación se ha realizado dentro del proyecto de investigación CS0201566260-C4-1-P Historia de la Programación y de los Programas de Televisión en España (cadenas de ámbito estatal): de la desregulación al apagón analógico, 19902010, financiado por el Ministerio de Economía y Competitividad (España).

\section{Referencias}

Batagelj, V. (2008). Analysis of Large Networks with Pajek. Networks, 22-27. Bignell, J. (2012). An Introduction to Television Studies. London: Routledge. https://doi.org/10.4324/9780203134955

Blondel, V. D., Guillaume, J. L., Lambiotte, R., \& Lefebvre, E. (2008). Fast unfolding of communities in large networks. Journal of Statistical Mechanics: Theory and Experiment, 10008-10020. https://doi.org/10.1088/1742-5468/2008/10/ P10008

Bondad-Brown, B. A., Rice, R. E., \& Pearce, K. E. (2012). Influences on TV Viewing and Online User-shared Video Use: Demographics, Generations, Contextual 
152 | Alicia Moreno-Delgado, Rafael Repiso y Julio Montero-Díaz

MONOGRÁFICO

Age, Media Use, Motivations, and Audience Activity. Journal of Broadcasting \& Electronic Media, 56(4), 471-493. https://doi.org/10.1080/08838151.2012 .732139

Brunsdon, C. (1998). What is the 'Television' of television studies? In D. Lusted \& C. Geraghty (Eds.), The Television Studies Book (pp. 95-113). London; New York.

Buckalew, J.K. (1969). News elements and selection by television news editors. Journal of Broadcasting, 14(1), 47-54. https://doi.org/10.1080/08838156909363575

Corner, J. (1999). Critical ideas in television studies. (C. Press, Ed.), Oxford television studies. New York: 0xford University Press.

Dow, B. J. (1996). Prime-time feminism : television, media culture, and the women's movement since 1970. University of Pennsylvania Press.

Espinar Ruiz, E. (2006). Imágenes y estereotipos de género en la programación $\mathrm{y}$ en la publicidad infantil. Análisis cuantitativo. Revista Latina de Comunicación Social, 61, 0. Retrieved from http://www.ull.es/publicaciones/ latina/200614Espinar_Ruiz.htm

Fiske, J. (1987). Television Culture. London; New York: Methuen.

Gabszewicz, J. J., Laussel, D., \& Sonnac, N. (2004). Programming and Advertising Competition in the Broadcasting Industry. Journal of Economics \& Management Strategy, 13(4), 657-669. https://doi.org/10.1111/j.1430-9134.2004.00027.x Hayama, T. (2018). Detecting TV Program Highlight Scenes Using Twitter Data Classified by Twitter User Behavior and Evaluating It to Soccer Game TV Programs. IEICE TRANSACTIONS ON INFORMATION AND SYSTEMS, E101D(4), 917924. https://doi.org/10.1587/transinf.2016IIP0020

Kamada, T., \& Kawai, S. (1988). A simple method for computing general position in displaying three-dimensional objects. Computer Vision, Graphics, and Image Processing, 41(1), 43-56. https://doi.org/10.1016/0734-189X(88)90116-8

Kotz, K., \& Story, M. (1994). Food advertisements during children's Saturday morning television programming: are they consistent with dietary recommendations? Journal of the American Dietetic Association, 94(11), 1296-1300. https://doi. org/10.1016/0002-8223(94)92463-5

Landhuis, C. E., Poulton, R., Welch, D., \& Hancox, R. J. (2008). Programming obesity and poor fitness: The long-term impact of childhood television. OBESITY, 16(6), 1457-1459. https://doi.org/10.1038/oby.2008.205 
Mastro, D. E., \& Behm-Morawitz, E. (2005). Latino Representation on Primetime Television. Journalism \& Mass Communication Quarterly, 82(1), 110-130. https://doi.org/10.1177/107769900508200108

Mastro, D. E., \& Greenberg, B. S. (2000). The Portrayal of Racial Minorities on Prime Time Television. Journal of Broadcasting \& Electronic Media, 44(4), 690-703. https://doi.org/10.1207/s15506878jobem4404_10

Montero-Díaz, J. (2014). Programación y programas de televisión en España antes de la desregulación (1956 1990) Introducción al monográfico. Estudios Sobre El Mensaje Periodístico, 20, 11-24.

Montero-Díaz, J. (2018). Una televisión con dos cadenas : la programación en España (1956-1990). Madrid: Cátedra.

Mulligan, D. A., Altmann, T. R., Brown, A., Christakis, D. A., Clarke-Pearson, K., Falik, H. L., ... Media, C. C. (2011). Policy Statement-Children, Adolescents, Obesity, and the Media. Pediatrics, 128(1), 201-208. https://doi.org/10.1542/ peds.2011-1066

Myers, P. N., \& Biocca, F. A. (1992). The elastic body-image - The effect of television advertising and programming on body-image distorsions in young-women. Journal of Communication, 42(3), 108-133. https://doi. org/10.1111/j.1460-2466.1992.tb00802.x

Orbe, M. P. (2008). Representations of Race in Reality TV: Watch and Discuss. Critical Studies in Media Communication, 25(4), 345-352. https://doi. org/10.1080/15295030802327790

Repiso, R., Ahedo, J., \& Montero, J. (2018). The presence of the encyclicals in Web of Science: a bibliometric approach. Scientometrics. https://doi.org/10.1007/ s11192-017-2636-z

Rozario, A. M., Masilamani, V., \& Arulchelvan, S. (2018). The case of the missing girls: distribution of gender roles in Indian children's television programming. Journal of Children and Media, 12(2), 125-142. https://doi.org/10.1080/1748 2798.2017.1423093

Savage, S. J., \& Wirth, M. (2005). Price, programming and potential competition in US cable television markets. Journal of Regulatory Economics, 27(1), 25-46. https://doi.org/10.1007/s11149-004-4417-x 
154 | Alicia Moreno-Delgado, Rafael Repiso y Julio Montero-Díaz MONOGRÁFICO

Stice, E., Schupak-Neuberg, E., Shaw, H. E., \& Stein, R. I. (1994). Relation of Media Exposure to Eating Disorder Symptomatology: An Examination of Mediating Mechanisms. Journal of Abnormal Psychology, 103(4), 836-840. https://doi. org/10.1037/0021-843X.103.4.836

Stice, E., \& Shaw, H. E. (1994). Adverse Effects of the Media Portrayed Thin-Ideal on Women and Linkages to Bulimic Symptomatology. Journal of Social and Clinical Psychology, 13(3), 288-308. https://doi.org/10.1521/jscp.1994.13.3.288

Taras, H. L., \& Gage, M. (1995). Advertised Foods on Children's Television. Archives of Pediatrics \& Adolescent Medicine, 149(6), 649-652. https://doi.org/10.1001/ archpedi.1995.02170190059010

Taras, H. L., Sallis, J. F., Patterson, T. L., Nader, P. R., \& Nelson, J. A. (1989). Television's influence on children's diet and physical activity. Journal of Developmental and Behavioral Pediatrics, 10(4), 176-180. https://doi. org/10.1097/00004703-198908000-00003

Turner, G., \& Tay, J. (2009). Television Studies After TV: Understanding Television in the Post-Broadcast Era. (G. Turner \& J. Tay, Eds.). Routledge.

Van Eck, N. J., \& Waltman, L. (2010). Software survey: VOSviewer , a computer program for bibliometric mapping. Scientometrics, (84), 523-538. https://doi. org/10.1007/s11192-009-0146-3

Wakshlag, J., \& Adams, W. J. (1985). Trends in program variety and the prime time access rule. Journal of Broadcasting \& Electronic Media, 29(1), 23-34. https:// doi.org/10.1080/08838158509386561

Williams, R. (1992). Television: Technology and Cultural Form (Routledge). London. Wilson, B. J., Smith, S. L., Potter, W. J., Kunkel, D., Linz, D., Colvin, C. M., \& Donnerstein, E. (2002). Violence in children's television programming: Assessing the risks. Journal of Communication, 52(1), 5-35. https://doi. org/10.1093/joc/52.1.5

\section{(9) 9}

Este obra está bajo una licencia de Creative Commons Reconocimiento 4.0 Internacional. 